\title{
NR2F2 regulates bone marrow-derived mesenchymal stem cell-promoted proliferation of Reh cells
}

\author{
NI ZHU* , HUAFANG WANG ${ }^{*}$, JIEPING WEI, BINSHENG WANG, WEI SHAN, \\ XIAOYU LAI, YANMIN ZHAO, JIAN YU and HE HUANG \\ Bone Marrow Transplantation Center, The First Affiliated Hospital, College of Medicine, \\ Zhejiang University, Hangzhou, Zhejiang 310003, P.R. China
}

Received July 1, 2015; Accepted April 21, 2016

DOI: $10.3892 / \mathrm{mmr} .2016 .5389$

\begin{abstract}
Bone marrow-derived mesenchymal stem cells (BM-MSCs) are pivotal components of the leukemic microenvironment. BM-MSCs have been previously reported to promote the proliferation of leukemic cells. To further understand the molecular mechanisms of BM-MSC-induced proliferation of leukemic cells, the present study co-cultured acute lymphoblastic leukemia (ALL) Reh cells with BM-MSCs. The current study used methods including shRNA, flow cytometry, MTT, reverse transcription-quantitative polymerase chain reaction, ELISA and western blotting. The data of the present study demonstrated that BM-MSCs promote the proliferation of Reh cells and the NR2F2 mRNA and protein levels were elevated in BM-MSCs following co-culture. Additionally, it was demonstrated that shRNA knockdown of NR2F2 inhibited BM-MSC-induced proliferation of Reh cells. Furthermore, following downregulation of NR2F2, vascular endothelial growth factor A (VEGFA) secretion by BM-MSCs was reduced. The present study demonstrated that NR2F2 mediates BM-MSC-induced proliferation of Reh cells, partially via regulation of VEGFA. Disrupting microenvironmental support by targeting NR2F2 may be a potential therapeutic strategy for ALL.
\end{abstract}

\section{Introduction}

Acute lymphoblastic leukemia (ALL) is a malignancy derived from cells of the lymphoid lineage. Despite good clinical outcomes for patients with childhood ALL, adult ALL remains clinically challenging due to a high rate of recurrence (1). It

Correspondence to: Professor He Huang, Bone Marrow Transplantation Center, The First Affiliated Hospital, College of Medicine, Zhejiang University, 79 Qingchun Road, Hangzhou, Zhejiang 310003, P.R. China

E-mail: huanghe@zju.edu.cn

*Contributed equally

Key words: NR2F2, mesenchymal stem cells, leukemic cells, proliferation, VEGFA has been previously reported that the overall 5-year survival rate for patients with ALL recurrence is 7\% (2). Previous studies have investigated the importance of the bone marrow microenvironment on ALL recurrence (3).

The bone marrow microenvironment is composed of various cell types and abundant extracellular matrix (4). Previous studies have demonstrated that stromal cells promote the proliferation and survival of leukemic cells by secreting soluble chemicals and adhesion molecules, including chemokine (C-X-C motif) ligand 12, IL (interleukin)-7 (5), vascular endothelial growth factor A (VEGFA), platelet derived growth factor (PDGF) (6), and via the integrin $\beta 1 /$ cluster of differentiation (CD) 44 axis (7). Stromal cell-induced proliferation may be associated with the development of minimal residual disease, which is considered to be an independent prognostic factor in ALL (8). Thus, it is necessary to investigate the mechanisms of bone marrow microenvironment-induced proliferation in leukemic cells. Mesenchymal stem cells (MSCs) are important components of the solid and hematologic tumor microenvironment (9). The present study investigated the bone marrow microenvironment-mediated proliferation of leukemic cells using bone marrow-derived (BM)-MSCs.

Nuclear receptor subfamily 2 group F member 2 (NR2F2) is a member of the nuclear receptor superfamily widely expressed in the mesenchymal compartment of developing organs (10). NR2F2 expression was previously reported to be abundant in MSCs and involved in the modulation of cell differentiation (11). NR2F2 has been predominantly investigated in solid malignancies, including ovarian and prostate cancer (12). Whether $\mathrm{NR} 2 \mathrm{~F} 2$ is important in the leukemic microenvironment remains to be investigated. Notably, the present study demonstrated that NR2F2 was upregulated in BM-MSCs following co-culture with Reh cells, which is a representative ALL cell line. The current study aimed to investigate the importance of NR2F2 in BM-MSC-induced proliferation of leukemic cells.

\section{Materials and methods}

Generation of BM-MSCs. Human bone marrow samples were collected from normal donors by bone marrow aspiration at The First Affiliated Hospital, College of Medicine, Zhejiang University (Hangzhou, China) and mononuclear cells were isolated by density gradient centrifugation at $400 \mathrm{x}$ g at $20^{\circ} \mathrm{C}$ 
for $25 \mathrm{~min}$. The current study was approved by the First Affiliated Hospital, College of Medicine, Zhejiang University. The cells were cultured in low-glucose Dulbecco's modified Eagle's medium (10-014-CVR; Corning Incorporated, Corning, NY, USA) supplemented with $10 \%$ fetal bovine serum (FBS; 10099-141; Gibco; Thermo Fisher Scientific, Inc., Waltham, MA, USA) at $37^{\circ} \mathrm{C}$ and $5 \% \mathrm{CO}_{2}$ in a humidified incubator. The medium was replaced after the initial $48 \mathrm{~h}$. Subsequently the medium was changed every 3 days. The adherent cells were passaged when $90 \%$ confluence was reached. BM-MSCs at passage 2-6 were used in the subsequent assays.

Characteristics of BM-MSCs. BM-MSCs of passages 3-5 were harvested using $0.25 \%$ trypsin-ethylenediaminetetraacetic acid solution (EDTA; 25200-056; Gibco; Thermo Fisher Scientific, Inc.). Cells were then incubated with the following anti-human monoclonal antibodies: CD90-fluorescein isothiocyanate (FITC; 1:20; 11-0909), CD105-phycoerythrin (PE; 1:20; 12-1057), CD73-APC (allophycocyanin; 1:20; 17-0739), CD45-FITC (1:20; 11-9459), CD34-PE (1:20; 12-0349), CD19-APC (1:20; 17-0199) and CD11b-PE (1:20; 12-0113) antibodies (eBioscience, Inc., San Diego, CA, USA) at $4^{\circ} \mathrm{C}$ for $30 \mathrm{~min}$. Following fixing with $75 \%$ ethanol (Sinopharm Chemical Reagent Co., Ltd.), permeabilisation with permeabilisation solution [CCS012A; Multi Sciences (Lianke) Biotech Co., Ltd., Hangzhou, China] and blocking with Human BD Fc Block (564219; BD Pharmingen, San Diego, CA, USA), cells were detected using an FC 500 MCL flow cytometer (Beckman Coulter, Inc., Brea, CA, USA) with mouse IgG1 $\mathrm{K}$ isotype control FITC (11-4714), mouse IgG1 K isotype control PE (12-4714), mouse IgG1 K isotype control APC (17-4714) isotype-matched antibodies (eBioscience, Inc.) applied as controls. BM-MSCs at passages 3-5 were cultured in osteogenic or adipogenic differentiation medium (HUXMA-90021 and -90031, respectively; Cyagen Biosciences, Inc., Santa Clara, CA, USA) for the differentiation assays. Cells were stained with Alizarin Red or Oil red O solution (HUXMA-90031; Cyagen Biosciences, Inc.) to evaluate osteogenic or adipogenic differentiation, respectively. The cells were analyzed using CXP Analysis Software 5 (Beckman Coulter, Inc.).

Cell culture and co-culture. The human ALL Reh cells were obtained from the Cell Bank of Shanghai Institute of Biochemistry and Cell Biology, Chinese Academy of Sciences (Shanghai, China), and were divided into cells cultured either alone or co-cultured with with BM-MSCs. Reh cells were cultured in Iscove's modified Dulbecco's medium (SH30228; Hyclone; GE Healthcare Life Sciences, Logan, UT, USA) supplemented with $10 \% \mathrm{FBS}$ at $37^{\circ} \mathrm{C}$ and $5 \% \mathrm{CO}_{2}$ in a humidified incubator. For co-culture experiments, BM-MSCs were initially seeded on 6 - or 12 -well plates at $5 \times 10^{4}$ cells $/ \mathrm{ml}$ density. After 4-6 h of culture, Reh cells were added to the adherent MSCs layer at $5 \times 10^{5}$ cells $/ \mathrm{ml}$ density. After $48 \mathrm{~h}$ of co-culture, Reh cells were collected by directly separating suspending cells as described by previous studies $(13,14)$.

Ki-67 detection by flow cytometry. Forkhead Box P3/Transcription Factor Staining Buffer Set (eBioscience, Inc.) was used for intracellular staining with the anti-human monoclonal Ki-67-PE antibody (12-5699; 1:20; eBioscience, Inc.). Reh cells were collected and washed with phosphate-buffered saline
(PBS) 2 times. Following incubation with fixation/permeabilization solution at $4^{\circ} \mathrm{C}$ for $30 \mathrm{~min}$, Reh cells were washed and incubated with $5 \mu \mathrm{l} \mathrm{Ki-67-PE} \mathrm{antibody} \mathrm{at} 4^{\circ} \mathrm{C}$ for $30 \mathrm{~min}$. Cells were resuspended in $500 \mu \mathrm{l}$ PBS for flow cytometric detection using an FC 500 MCL flow cytometer. Ki-67 was detectable during the active phases of cell cycle $\left(\mathrm{G}_{1}, \mathrm{~S}, \mathrm{G}_{2}\right.$ and $\left.\mathrm{M}\right)$ and not during the resting phase $\left(\mathrm{G}_{0}\right)$, therefore, the positive cells were considered to be proliferating cells (15).

Apoptosis assay. Reh cells were harvested and washed twice with cold PBS. Cells were resuspended in $100 \mu \mathrm{l} 1 \mathrm{X}$ binding buffer provided in the Apoptosis Detection kit (559763; BD Pharmingen, San Diego, CA, USA). Following incubation with the Annexin V-PE antibody and 7-aminoactinomycin D from the Apoptosis Detection kit (559763; BD Pharmingen) at room temperature for $15 \mathrm{~min}$, Reh cells were analyzed using the FC 500 MCL flow cytometer. Cells that were Annexin V-positive were considered to be apoptotic.

Cell cycle assay. Reh cells were fixed with chilled $75 \%$ ethanol for $2 \mathrm{~h}$ at $-20^{\circ} \mathrm{C}$. Following rehydration in PBS for $15 \mathrm{~min}$, cells were washed twice and resuspended in $1 \mathrm{ml}$ propidium iodide (CCS012A; Multi Sciences Biotech Co., Ltd., Hangzhou, China), then incubated at room temperature for $30 \mathrm{~min}$. Samples were analyzed using the FC 500 MCL flow cytometer. Data were analyzed using MultiCycle software, version 3.2 (Beckman Coulter, Inc.).

Knock-down of NR2F2. A previously reported (16), NR2F2-targeting shRNA (5'-AGGTAACGTGATTGA TTCAGTATCTTA-3') was cloned into the pGLV3 plasmid (Genepharm, Inc., Sunnyvale, CA, USA). A scrambled sequence (5'-TTCTCCGAACGTGTCACGT-3') was also cloned into the pGLV3 as a negative control. shRNAs were obtained from Shanghai GenePharma Co., Ltd. (Shanghai, China). Highly concentrated lentivirus was produced by co-transfecting 293T cells (Cell Bank of Shanghai Institute of Biochemistry and Cell Biology, Chinese Academy of Sciences) with pMD2.G (Thermo Fisher Scientific, Inc.), psPAX2 (Thermo Fisher Scientific, Inc.) and shRNA plasmids with Lipofectin (Thermo Fisher Scientific, Inc.) according to the manufacturer's instructions. The viral suspension was collected, filtered and used to transfect BM-MSCs directly following removal of medium. After $10 \mathrm{~h}$, virus-containing medium was replaced with fresh medium. The positive expression of green fluorescent protein was assessed by fluorescent microscopy (Eclipse-Ti K11301; Nikon Corporation, Tokyo, Japan) and used to evaluate transfection efficiency.

Cell viability assay. Transfected BM-MSCs were seeded onto a 96-well plate at a concentration of $5 \times 10^{3}$ cells/well. Five parallel wells of cells were seeded for each group (knockdown BM-MSCs group and negative control BM-MSCs group). Following incubation for $48 \mathrm{~h}, 10 \mu \mathrm{l}$ 3-(4,5-dimethyl-2-thiazolyl) 2,5-diphenyltetrazolium bromide (MTT; LK-MTT001; Hangzhou Lianke Biology Technology Ltd., Co., Hangzhou, China) was added to all wells. Medium was discarded after $4 \mathrm{~h}$. The optical density (OD) values were evaluated by the SpectraMax M5 microplate reader (Molecular Devices, LLC, Sunnyvale, CA, USA) at $570 \mathrm{~nm}$ following addition of $100 \mu \mathrm{l}$ dimethyl sulfoxide (Sinopharm Chemical Reagent Co., Ltd., Shanghai, China). 
A
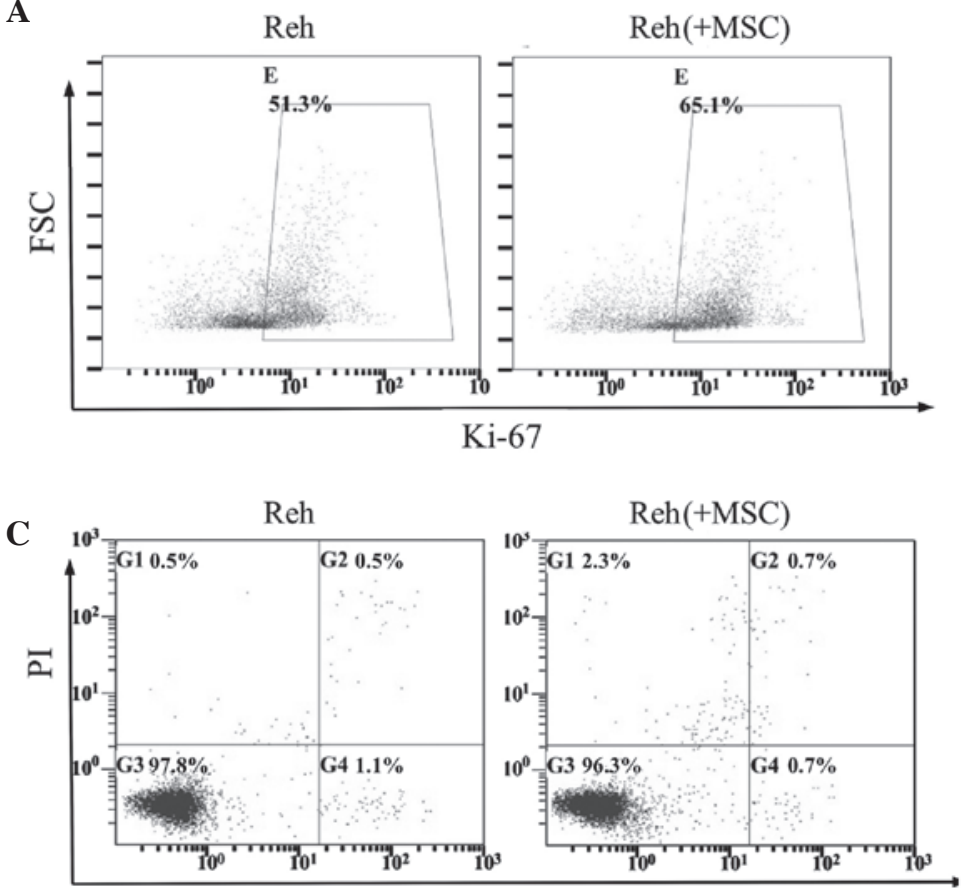

Annexin V-FITC
B

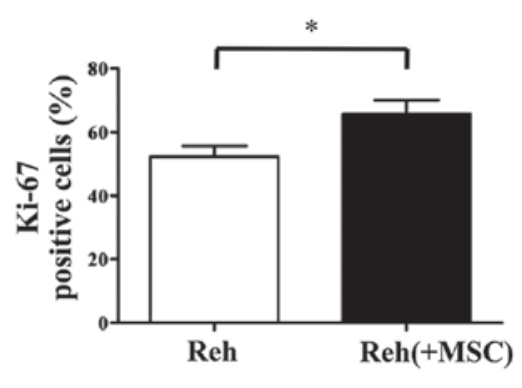

D

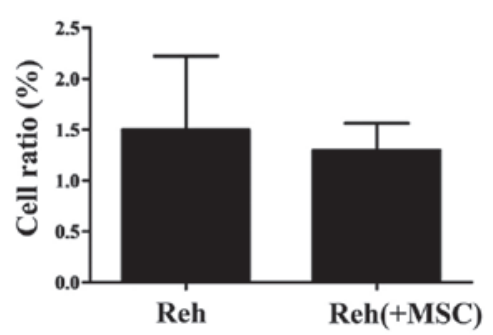

Figure 1. BM-MSCs promote the proliferation of Reh cells, however have no observable effect on apoptosis. Reh cells were cultured alone or with BM-MSCs for 48 h. (A) Representative graphs of Ki-67 antigen staining detected by flow cytometry. (B) Percentage of Ki-67-positive cells in Reh cells or Reh cultured with BM-MScs. (C) Representative graphs of apoptosis detected by flow cytometry. (D) Percentage of apoptotic cells in the groups of Reh cells cultured alone or with BM-MSCs. Values are presented as the mean \pm standard deviation. "P<0.05. MSC, mesenchymal stem cell; BM-MSC, bone marrow-derived MSC; FSC, forward scatter; Ki-67, marker of proliferation Ki-67; FITC, fluorescein isothiocyanate; PI, propidium iodide.

Enzyme-linked immunosorbent assay (ELISA). The concentration of VEGFA in the co-culture medium was assessed using an ELISA kit (BMS277/2; eBioscience, Inc.). Briefly, co-culture medium supernatant was collected at 24 or $48 \mathrm{~h}$ following separation of Reh cells by concentration using centrifugation at $300 \mathrm{x} \mathrm{g}$ at $20^{\circ} \mathrm{C}$ for $10 \mathrm{~min}$. An adequate volume of sample was incubated with pre-coated plates according to the manufacturer's protocol. The OD value was detected at $450 \mathrm{~nm}$ using the SpectraMax M5 microplate reader. Standard curves were produced using the standards of known concentration supplied in the kit and used to calculate protein concentrations.

Reverse transcription-quantitative polymerase chain reaction (RT-qPCR). Co-cultured BM-MSCs were isolated by thoroughly washing with PBS until all Reh cells were removed. Total cellular RNA was extracted from co-cultured BM-MSCs using TRIzol reagent (15596-026; Thermo Fisher Scientific, Inc.) PrimeScript RT reagent kit with gDNA Eraser (RR047A; Takara Biotechnology Co., Ltd., Dalian, China) was used for RT. The RT protocol was as follows: $37^{\circ} \mathrm{C}$ for $15 \mathrm{~min} ; 85^{\circ} \mathrm{C}$ for $5 \mathrm{sec}$; stored at $4^{\circ} \mathrm{C}$. qPCR was performed using SYBR Premix Ex Taq II (RR420A; Takara Biotechnology Co., Ltd.) according to manufacturer's instructions in a LightCycler system (Roche Diagnostics, Basel, Switzerland). Each reaction was performed in triplicate. The cycling conditions were as follows: Initial denaturation at $95^{\circ} \mathrm{C}$ for $5 \mathrm{~min}, 45$ cycles of $95^{\circ} \mathrm{C}$ for $5 \mathrm{sec}$ and $60^{\circ} \mathrm{C}$ for $20 \mathrm{sec}$, melting curve analysis at $95^{\circ} \mathrm{C}$ for $15 \mathrm{sec}, 65^{\circ} \mathrm{C}$ for $15 \mathrm{sec}$ and then maintained at $95^{\circ} \mathrm{C}$. The expression of glyceraldehyde 3-phophate dehydrogenase (GAPDH) was used as a reference gene. The primers used were as follows: NR2F2, forward 5'-CAACCAGCCGAC GAGATT-3' and reverse 5'-ATTGCTCTATGACTGAGG AGGAGAC-3'; VEGFA, forward 5'-ACAGGTACAGGGATG AGGACAC-3' and reverse 5'-AAGCAGGTGAGAGTAAGC GAAG-3'; GAPDH, forward 5'-AGAAGGCTGGGGCTC ATTTG-3' and reverse 5'-AGGGGCCATCCACAGTCTTC-3'.

Western blot analysis. The transfected BM-MSCs were harvested by digesting using $0.25 \%$ trypsin-EDTA solution. Cells were lysed on ice for $1 \mathrm{~h}$ in radioimmunoprecipitation assay lysis buffer (AR0105; Wuhan Boster Biological Technology, Ltd., Wuhan, China) with phenylmethylsulfonyl fluoride solution (AR1179; Wuhan Boster Biological Technology, Ltd.). Cell lysates were centrifuged at $12,000 \mathrm{x}$ g for $5 \mathrm{~min}$ at $4^{\circ} \mathrm{C}$. The supernatant was incubated at $100^{\circ} \mathrm{C}$ for $10 \mathrm{~min}$ following addition of an appropriate volume of loading buffer (5-fold dilution; Hangzhou Fu De Biological Technology, Co., Ltd., Hangzhou, China). Samples were loaded onto a $10 \%$ polyacrylamide gels containing sodium dodecyl sulfate (Hangzhou Fu De Biological Technology, Co., Ltd.), electrophoretically separated (110 V for $100 \mathrm{~min}$ ) and transferred onto a nitrocellulose membrane. Membranes were blocked at $20^{\circ} \mathrm{C}$ for $2 \mathrm{~h}$ with $5 \%$ non-fat milk then incubated with the primary antibodies at $4^{\circ} \mathrm{C}$ overnight, and then incubated with fluorescent IgG IRDye ${ }^{\circledR} 650$ goat anti-mouse (1:10,000; 926-65010) and goat anti-rabbit (1:10,000; 926-65020) secondary antibodies (LI-COR, Inc., Lincoln, NE, USA) at room temperature for $1 \mathrm{~h}$. Rabbit monoclonal NR2F2 $(1: 1,000 ; \# 6434)$ and mouse monoclonal GAPDH $(1: 1,000$; \#5079) primary antibodies were purchased from Cell Signaling Technology, Inc. (Danvers, MA, USA) and Multi Sciences 
A
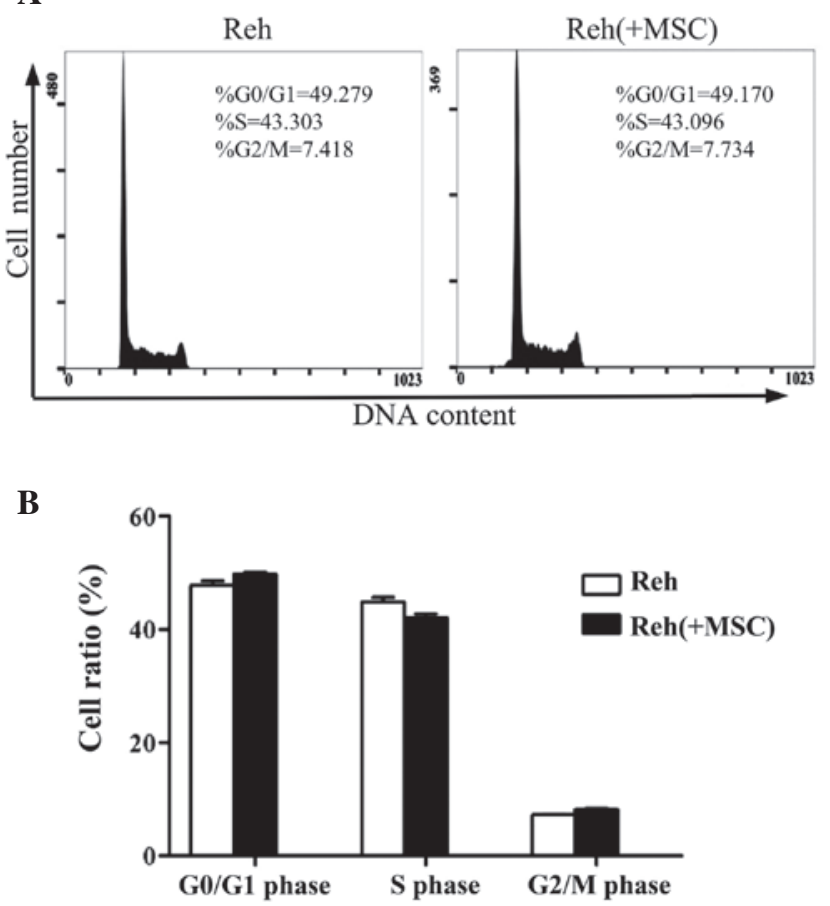

Figure 2. Co-culture with BM-MSCs increases the percentage of Reh cells in the $\mathrm{G}_{2} / \mathrm{M}$ phase. (A) Representative graphs of the percentage cells in the cell cycle phases detected by flow cytometry in Reh cells cultured alone or with BM-MSCs for $48 \mathrm{~h}$. (B) The mean \pm standard deviation of the percentages of Reh cells in different cell cycle phases when cultured alone or with BM-MSCs. BM-MSC, bone marrow-derived mesenchymal stem cell.

(Lianke) Biotech Co., Ltd., respectively. Immunoreactive bands were visualized using an Odyssey Infrared Imaging System (LI-COR, Inc.). GAPDH was used as a loading control.

Statistical analysis. All independent experiments were repeated $\geq 3$ times. Statistical analysis was performed using SPSS software, version 19.0 (IBM SPSS, Armonk, NY, USA). Differences between mean values were evaluated using Student's two-tailed t-test. Values are presented as the mean \pm standard deviation and $\mathrm{P}<0.05$ was considered to indicate a statistically significant difference.

\section{Results}

Characteristics of BM-MSCs. BM-MSCs exhibited a spindle-like shape and fibroblast-like morphology. BM-MSCs were CD90and CD105-positive, and predominantly CD73-positive. However, the cells were negative for CD19 and CD11b, and hematopoietic markers, including CD34 and CD45. The characteristics of BM-MSCs have been described in a previous report (17).

BM-MSCs promote the proliferation of Reh cells. In order to assess whether BM-MSCs promote Reh cell proliferation, Reh cells were isolated and the expression of the Ki-67 antigen was analyzed. As demonstrated in Fig. 1A and B, Reh cells that were co-cultured with BM-MSCs for $48 \mathrm{~h}$ exhibited a significantly increased percentage of Ki-67-positive cells compared with Reh cells cultured alone $(\mathrm{P}<0.05)$. The present study also assessed whether BM-MSCs affected the apoptosis of Reh cells. Co-culture with BM-MSCs for $48 \mathrm{~h}$ had no observable effect
A
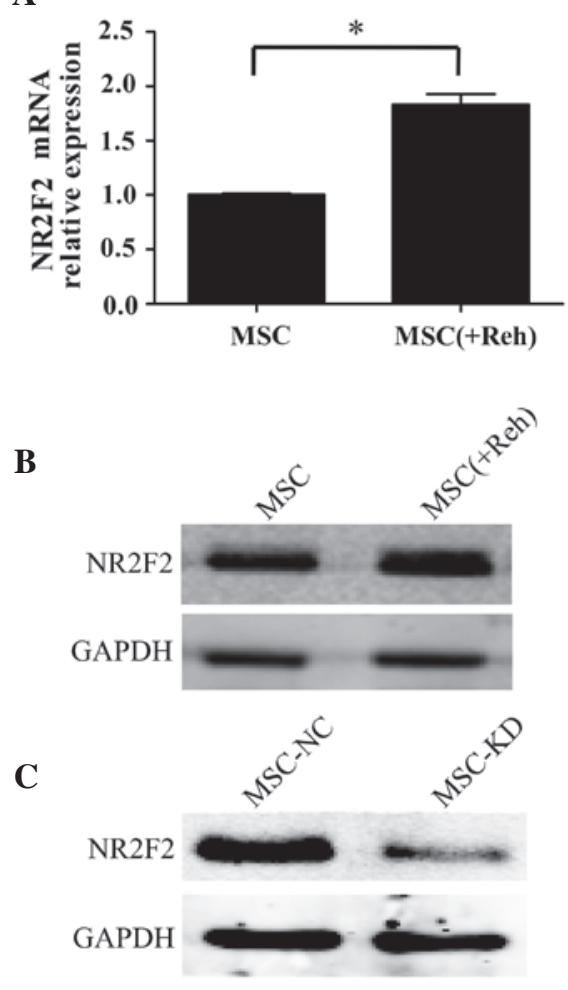

Figure 3. Levels of NR2F2 are upregulated in BM-MSCs following co-culture with Reh cells. (A) NR2F2 mRNA expression levels in BM-MSCs were measured following culture alone or co-culture with Reh cells for $48 \mathrm{~h}$. Values are presented as the mean \pm standard deviation. ${ }^{*} \mathrm{P}<0.05$. (B) The protein expression levels of NR2F2 in BM-MSCs cultured alone or co-cultured with Reh cells for $48 \mathrm{~h}$ were analyzed by western blotting. (C) The knockdown of NR2F2 using short hairpin RNA was confirmed by western blot analysis. NR2F2, nuclear receptor subfamily 2 group F member 2; BM-MSC, bone marrow-derived mesenchymal stem cell; NC, negative control; KD, NR2F2 knockdown.

on the apoptosis of Reh cells (Fig. 1C and D). Additionally, the cell cycle pattern of Reh cells was analyzed in the co-culture system. When co-cultured with BM-MSCs, the percentage of Reh cells in the $\mathrm{G}_{2} / \mathrm{M}$ phase was markedly increased compared with Reh cells cultured alone. Whereas the percentages of cells in $\mathrm{G}_{0} / \mathrm{G}_{1}$ or $\mathrm{S}$ phases were not significantly different (Fig. 2).

NR2F2 regulates the BM-MSCs-promoted proliferation of Reh cells. To analyze the expression levels of NR2F2 in BM-MSCs, BM-MSCs were separated from Reh cells following co-culture for $48 \mathrm{~h}$. The mRNA and protein levels of NR2F2 in BM-MSCs were observed to be upregulated when co-cultured with Reh cells compared with culture alone (Fig. 3A and B). In order to further elucidate whether NR2F2 is involved in mediating the BM-MSC-induced proliferation of Reh cells, NR2F2 expression was measured in BM-MSCs, and was identified to be downregulated by transfection of an NR2F2 antisense shRNA. Western blot analysis demonstrated that the expression of NR2F2 was significantly knocked down by the shRNA (Fig. 3C). As demonstrated in Fig. 4A and B, the percentage of Ki-67-positive Reh cells in the NR2F2 knockdown BM-MSC co-culture group was significantly reduced compared with the negative control group $(\mathrm{P}<0.05)$. Furthermore, the percentage of Ki-67-positive Reh cells in the NR2F2 knockdown BM-MSC co-culture group was similar to the percentage observed when Reh cells were 
A

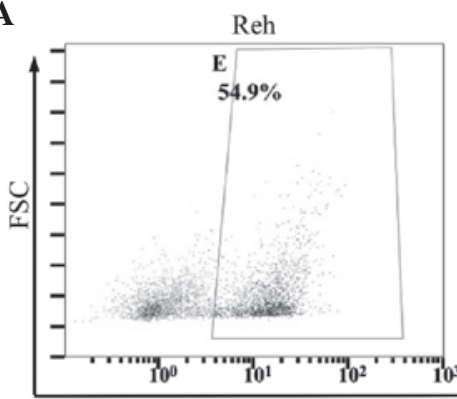

B

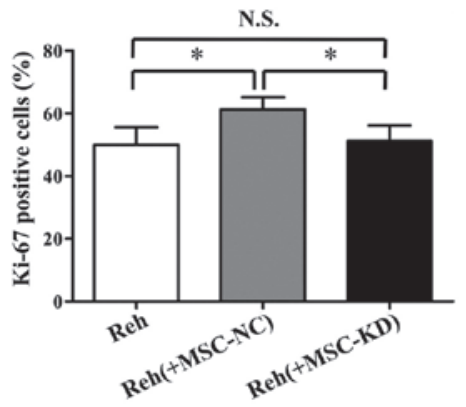

$\operatorname{Reh}(+\mathrm{MSC}-\mathrm{NC})$

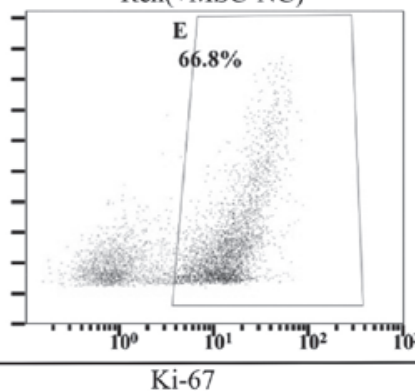

$\operatorname{Reh}(+\mathrm{MSC}-\mathrm{KD})$

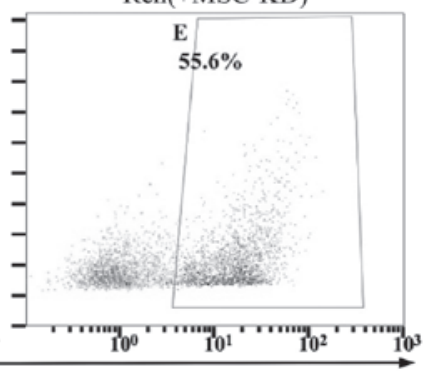

C

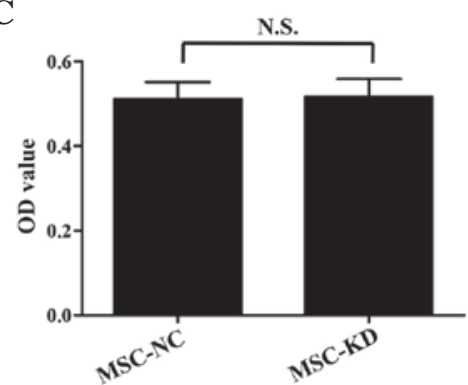

Figure 4. Downregulation of NR2F2 inhibits BM-MSCs-mediated increased proliferation of Reh cells. (A) Representative graphs of Ki-67 antigen staining detected by flow cytometry in Reh cells cultured alone or with BM-MSCs, including NC group and NR2F2-KD group. (B) Percentage of Ki-67-positive cells are presented as the mean \pm standard deviation in the three groups. (C) The OD values at $570 \mathrm{~nm}$ in BM-MSC NC and NR2F2-KD groups are presented as the mean \pm standard deviation. ${ }^{*} \mathrm{P}<0.05$. FSC, forward scatter; BM-MSC, bone marrow-derived mesenchymal stem cell; NC, negative control; KD knockdown.
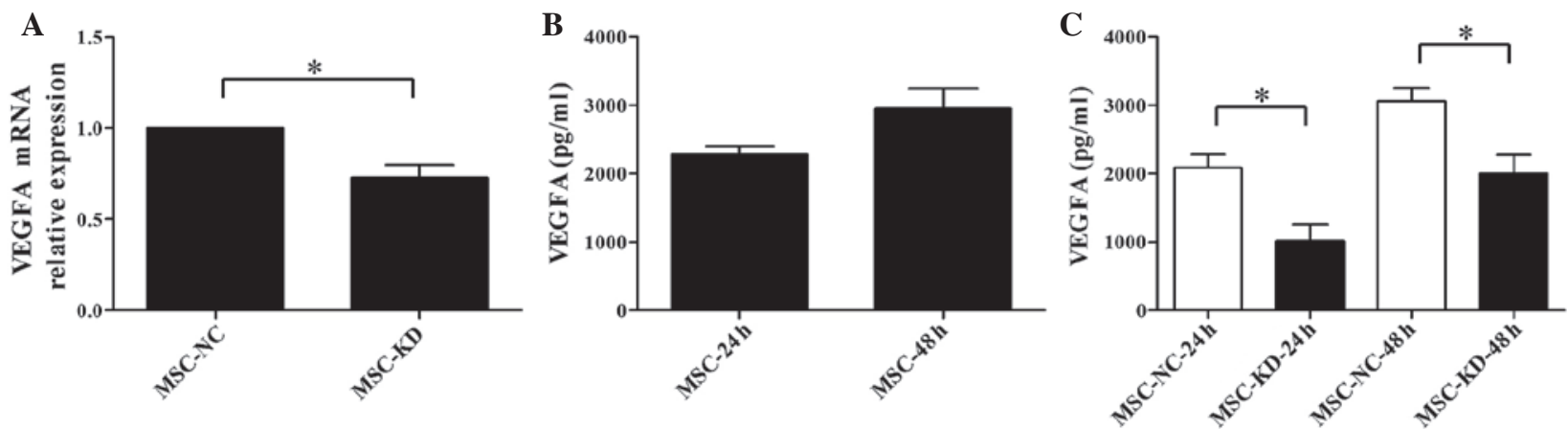

Figure 5. Down regulation of NR2F2 suppresses the expression of VEGFA in BM-MSCs. Reh cells and BM-MSCs were co-cultured for 24 and $48 \mathrm{~h}$, and additionally NR2F2 was knocked down in BM-MSCs. (A) Relative VEGFA mRNA expression levels in the BM-MSC NC and NR2F2-KD groups were measured by reverse transcription-quantitative polymerase chain reaction following $48 \mathrm{~h}$ in co-culture with Reh cells. (B) The protein concentration of VEGFA in the co-culture supernatant of untransfected BM-MSCs was measured at 24 and $48 \mathrm{~h}$. (C) VEGFA concentration was detected in the co-culture supernatant of the $\mathrm{NC}$ and $\mathrm{KD}$ groups at 24 and $48 \mathrm{~h}$. Values are presented as the mean \pm standard deviation. "P<0.05. NR2F2, nuclear receptor subfamily 2 group $\mathrm{F}$ member 2 ; VEGFA, vascular endothelial growth factor A; BM-MSC, bone marrow-derived mesenchymal stem cell; NC, negative control; KD, NR2F2 knockdown.

cultured alone. Additionally, the cell viabilities of BM-MSCs in the knockdown and control groups were compared to exclude the possibility that silencing NR2F2 affected the cell viability. As demonstrated by the MTT assay, no significant difference in cell viability was observed between the two groups (Fig. 4C). These data suggested that NR2F2 is involved in the regulation of BM-MSC-induced proliferation of Reh cells.

Downregulation of NR2F2 suppresses the expression of $V E G F A$ in BM-MSCs. As it has been previously reported that VEGFA provides important proliferative cues for ALL cells in a BM-MSC-conditioned system (6), the present study attempted to evaluate whether the effects of NR2F2 on the co-culture system were mediated by VEGFA. BM-MSCs were isolated from the co-culture after $48 \mathrm{~h}$ and the mRNA expression levels of VEGFA were measured in NR2F2 knockdown and negative control cells. Following NR2F2 knockdown, VEGFA was significantly downregulated $(\mathrm{P}<0.05$; Fig. 5A). To further investigate the association between NR2F2 and VEGFA, the concentration of VEGFA in the co-culture supernatant was measured by ELISA at 24 and $48 \mathrm{~h}$. A relatively high concentration of VEGFA was detected in the supernatant of BM-MSCs (Fig. 5B). However, the concentration of VEGFA was barely detectable in the supernatant of Reh cells cultured alone (data not shown). The concentration of VEGFA was significantly reduced in the NR2F2-knockdown group compared with the negative control at 24 and $48 \mathrm{~h}(\mathrm{P}<0.05$; Fig. 5C). Thus, these results demonstrated that VEGFA is involved in the mediation of the effects of NR2F2 on BM-MSC-induced proliferation of Reh cells. 


\section{Discussion}

The proliferation and survival of ALL cells has been previously reported to be enhanced by stromal cells (6). The present study demonstrated that following co-culture with BM-MSCs, the percentage of Ki-67 antigen-positive Reh cells was increased, demonstrating that BM-MSCs induced Reh cell proliferation. Additionally, in accordance with a previous study (18), apoptosis was measured and no significant differences were observed between Reh cells cultured alone or those co-cultured with BM-MSCs. Yang et al (13) reported that MSCs induce the proliferation of Reh cells and demonstrated that the percentage of Reh cells in the $S$ and $\mathrm{G}_{2} / \mathrm{M}$ phases was increased in co-culture conditions. The present study only observed an increased percentage of Reh cells in $\mathrm{G}_{2} / \mathrm{M}$ phase, no observable changes in the percentage of cells in $\mathrm{S}$ phase were detected. Although the data of the present study is different to that of a previous study (13), this may be explained by the fact that the cell cycle assay used in the present study did not distinguish between the $\mathrm{G}_{0}$ and $\mathrm{G}_{1}$ phases.

Previous studies have demonstrated that stromal cell-mediated increases in proliferation are primarily caused by cytokines produced by stromal cells, including IL-6, VEGFA and PDGF (6). However, the mechanisms involved in producing these cytokines remain unclear. The data of the present study indicated that NR2F2 levels are upregulated in BM-MSCs co-cultured with Reh cells. Furthermore, downregulation of NR2F2 with shRNA attenuated BM-MSC-induced proliferation of Reh cells, which demonstrates that NR2F2 is involved in this process. The present study also demonstrated that knockdown of NR2F2 did not affect the viability of BM-MSCs after $48 \mathrm{~h}$ culture. Therefore, the mechanism of NR2F2-regulated BM-MSC-induced proliferation is not associated with effects on cell viability.

NR2F2 has been previously reported to regulate VEGF/VEGF receptor 2 signaling in endothelial cells (19), thus, the present study hypothesized that NR2F2 participates in the regulation of VEGFA in the co-culture system. The results of RT-qPCR and ELISA assays demonstrated that downregulation of NR2F2 in BM-MSCs inhibited the production of VEGFA. Further research is required to explore the specific mechanisms that mediate the effect of NR2F2 on the production of VEGFA in BM-MSCs. The present study additionally demonstrated that VEGFA is secreted by BM-MSCs and detected in the culture supernatant. However, VEGFA was not detected in the supernatant of Reh cells cultured alone. These data suggest that BM-MSC-induced proliferation of Reh cells is partially mediated by the secretion of VEGFA.

In conclusion, the present study demonstrated that BM-MSCs promote the proliferation of Reh cells and that this process was partially mediated by NR2F2 regulation of VEGFA expression. Targeting NR2F2 may be a potential therapeutic strategy to inhibit the proliferation of ALL cells by disrupting the microenvironmental support.

\section{Acknowledgements}

The current study was supported by the National Natural Science Foundation of China (grant nos. 81230014, 81100364 and 81370647) and the Natural Science Foundation of Zhejiang Province (grant no. Y13H080008).

\section{References}

1. Faderl S, O'Brien S, Pui CH, Stock W, Wetzler M, Hoelzer D and Kantarjian HM: Adult acute lymphoblastic leukemia: Concepts and strategies. Cancer 116: 1165-1176, 2010.

2. Fielding AK, Richards SM, Chopra R, Lazarus HM, Litzow MR, Buck G, Durrant IJ, Luger SM, Marks DI, Franklin IM, et al; Medical Research Council of the United Kingdom Adult ALL Working Party; Eastern Cooperative Oncology Group: Outcome of 609 adults after relapse of acute lymphoblastic leukemia (ALL); an MRC UKALL12/ECOG 2993 study. Blood 109: 944-950, 2007.

3. Shishido S, Bönig H and Kim YM: Role of integrin alpha4 in drug resistance of leukemia. Front Oncol 4: 99, 2014.

4. Clark BR and Keating A: Biology of bone marrow stroma. Ann N Y Acad Sci 770: 70-78, 1995.

5. Juarez J, Baraz R, Gaundar S, Bradstock K and Bendall L: Interaction of interleukin-7 and interleukin-3 with the CXCL12-induced proliferation of B-cell progenitor acute lymphoblastic leukemia. Haematologica 92: 450-459, 2007.

6. Gaundar SS, Bradstock KF and Bendall LJ: p38MAPK inhibitors attenuate cytokine production by bone marrow stromal cells and reduce stroma-mediated proliferation of acute lymphoblastic leukemia cells. Cell Cycle 8: 2975-2983, 2009.

7. Malfuson JV, Boutin L, Clay D, Thépenier C, Desterke C, Torossian F, Guerton B, Anginot A, de Revel T, Lataillade JJ and Le Bousse-Kerdilès MC: SP/drug efflux functionality of hematopoietic progenitors is controlled by mesenchymal niche through VLA-4/CD44 axis. Leukemia 28: 853-864, 2014.

8. van Dongen JJ, van der Velden VH, Brüggemann M and Orfao A: Minimal residual disease diagnostics in acute lymphoblastic leukemia: Need for sensitive, fast, and standardized technologies. Blood 125: 3996-4009, 2015.

9. Casey SC, Vaccari M, Al-Mulla F, Al-Temaimi R, Amedei A, Barcellos-Hoff MH, Brown DG, Chapellier M, Christopher J, Curran CS, et al: The effect of environmental chemicals on the tumor microenvironment. Carcinogenesis 36 (Suppl 1): S160-S183, 2015

10. Pereira FA, Qiu Y, Zhou G, Tsai MJ and Tsai SY: The orphan nuclear receptor COUP-TFII is required for angiogenesis and heart development. Genes Dev 13: 1037-1049, 1999.

11. Xie X, Qin J, Lin SH, Tsai SY and Tsai MJ: Nuclear receptor chicken ovalbumin upstream promoter-transcription factor II (COUP-TFII) modulates mesenchymal cell commitment and differentiation. Proc Natl Acad Sci USA 108: 14843-14848, 2011.

12. Safe S, Jin UH, Hedrick E, Reeder A and Lee SO: Minireview: Role of orphan nuclear receptors in cancer and potential as drug targets. Mol Endocrinol 28: 157-172, 2014.

13. Yang Y, Mallampati S, Sun B, Zhang J, Kim SB, Lee JS, Gong Y, Cai $Z$ and Sun $X$ : Wnt pathway contributes to the protection by bone marrow stromal cells of acute lymphoblastic leukemia cells and is a potential therapeutic target. Cancer Lett 333: 9-17, 2013.

14. Hu K, Gu Y, Lou L, Liu L, Hu Y, Wang B, Luo Y, Shi J, $\mathrm{Yu} \mathrm{X}$ and Huang $\mathrm{H}$ : Galectin-3 mediates bone marrow microenvironment-induced drug resistance in acute leukemia cells via Wnt/ $\beta$-catenin signaling pathway. J Hematol Oncol 8: 1, 2015.

15. Beresford MJ, Wilson GD and Makris A: Measuring proliferation in breast cancer: Practicalities and applications. Breast Cancer Res 8: 216, 2006.

16. Li L, Xie X, Qin J, Jeha GS, Saha PK, Yan J, Haueter CM, Chan L, Tsai SY and Tsai MJ: The nuclear orphan receptor COUP-TFII plays an essential role in adipogenesis, glucose homeostasis, and energy metabolism. Cell Metab 9: 77-87, 2009.

17. Wang B, Hu Y, Liu L, Hu K, Tie R, He Y, Fu S, Zhu N, Luo Y, Yu X and Huang H: Phenotypical and functional characterization of bone marrow mesenchymal stem cells in patients with chronic graft-versus-host disease. Biol Blood Marrow Transplant 21: 1020-1028, 2015.

18. Zhang Y, Hu K, Hu Y, Liu L, Wang B and Huang H: Bone marrow mesenchymal stromal cells affect the cell cycle arrest effect of genotoxic agents on acute lymphocytic leukemia cells via p21 down-regulation. Ann Hematol 93: 1499-1508, 2014.

19. Chen X, Qin J, Cheng CM, Tsai MJ and Tsai SY: COUP-TFII is a major regulator of cell cycle and Notch signaling pathways. Mol Endocrinol 26: 1268-1277, 2012. 